\title{
LEARNING AND DEVELOPMENT OF THYROID FNA IN A ENDOCRINE CLINIC
}

Lorenzo J, Boente R, Antón $\mathrm{I}^{(1)}$ and Sas M. Endocrinology and Pathologic ${ }^{(1)}$ department Hospital Povisa. Vigo.Spain

Background: Development of Thyroid Ultrasound and FNA is more likely to make it in a endocrine clinic, that means to make more diagnosis medical process and so to avoid more outpatients visits. But it's necessary a specific training. We tried to evaluated our training. Material y methods: We have checked FNAs developed in our clinic in the last two and half year. After specific training in radiologic department, we have compared our results in two consecutive periods. The first one was in the first year after training developing FNAs and the second period was in the next one and half year with FNAs. (in the second period we used Bethesda system)

Results: 322 FNAs have been made in our clinic. (83,8\% diagnosis and 16,2\% non diagnosis). In the first phase (one year) we made 102 FNAs with 25 (24,5\%) non diagnosis and 77 (75,5\%) diagnosis, results are exhibited. In the second phase (one and half year) 220 FNAs were made and: $27(12,27 \%)$ non diagnosis and $193(87,73 \%)$ diagnosis. Specific FNAs results are exhibited too. (Bethesda system: benign: 166, atipic:14, suspicious neo follicular and Hurthle: 16, suspicius of malignant: 6 , medullar:1).

Conclusions: FNAs thecnique development after period learning is possible in a endocrine clinic with satisfactory results, similar to others published series. Its useful to patient because less visits to hospital, more efficient in your clinic activity and cheaper medical process. Bethesda system make easier comunication between clinics and pathologics. 